\title{
DISTRIBUTED AND CONCURRENT OBJECTS BASED ON LINEAR LOGIC
}

Naoki Kobayashi and Akinori Yonezawa

\author{
Department of Information Science \\ University of Tokyo \\ \{koba,yonezawa\} @is.s.u-tokyo.ac.jp
}

\begin{abstract}
There are recently growing interests in distributed/mobile applications. Specification of such applications requires consideration of some specific features that were not present in single processor applications: migration of data and computation, locationdependence, etc. Objects are attractive devices for describing such applications because an object gives a natural unit of migration and the location dependence can be expressed by binding the same name to different objects on different locations.

One way for modeling such distributed objects would be to take what we call the process calculi approach $[1,3,9]$, in which the syntax for expressing migration and location dependence is introduced and then its precise operational semantics is defined using transition systems. This approach can specify the precise behavior of distributed objects to the extent that their implementation on actual machines is fairly clear. On the other hand, such approach is often likely to overspecify the desired behavior of objects. For example, suppose we want to express computation that invokes methods of two remote objects and gathers the result. From an operational viewpoint, we can specify such computation either as a migrating object that moves to the location of each object and invokes the methods locally, and comes back to the original location, or as an object that stays at current location and remotely invokes methods of the objects. However, if we are only interested in the result of computation, both objects can be considered to reveal the same behavior. Although process calculi usually emphasize some process equivalence theories, it is often cumbersome to actually check the equivalence of two processes.

In this work, we would rather take a different approach: we use linear logic for expressing distributed objects. Linear logic [4] has been successfully used for specifying concurrent processes/objects $[2,8,6]$. Following such success, we use
\end{abstract}


here some extensions of linear logic $[7,5]$ and show how the behavior of many kinds of distributed objects can be specified. As was already observed in [7], we can elegantly express object migration and location dependence by using modal operators. One advantage of our approach is that it can give a more abstract specification of distributed object behavior than an operational approach: indeed, the two objects in the above example can be expressed as logically equivalent formulas of a modal linear logic, hence they are not distinguished. Of course, this fact can also be considered a disadvantage: we cannot specify the exact operational behavior of distributed objects. As discussed in [7], when we express objects as formulas of the modal linear logic, the logical equivalence implies only may equivalence of object behaviors. We can, however, remedy this problem by further extending the underlying linear logic with temporal operators such as those introduced in [5]. Thus, by choosing a certain kind of linear logic, we can specify the behavior of distributed objects with an appropriate degree of preciseness.

\section{References}

[1] R. Amadio and S. Prasad. "Localities and failures (extended summary)", in Proceedings of 14th FST and TCS Conference, FST-TCS'94, volume 880 of Lecture Notes in Computer Science, pages 205-216, 1994.

[2] J.-M. Andreoli and R. Pareschi. "Linear objects: Logical processes with built-in inheritance", New Generation Computing, 9:445-473, 1991.

[3] C. Fournet, G. Gonthier, J. Lévy, L. Maranget, and D. Rémy. "A calculus of mobile agents", in Proceedings of CONCUR'96, volume 1119 of Lecture Notes in Computer Science, pages 406-421. Springer-Verlag, 1996.

[4] J.-Y. Girard. "Linear logic", Theoretical Computer Science, 50:1-102, 1987.

[5] M. Kanovich and T. Ito. "Temporal linear logic specifications for concurrent processes", in Proceedings of IEEE Symposium on Logic in Computer Science, pages 48-57, 1997.

[6] N. Kobayashi. "Concurrent linear logic programming", $\mathrm{PhD}$ Thesis, Department of Information Science, University of Tokyo, April 1996.

[7] N. Kobayashi, T. Shimizu, and A. Yonezawa. "Distributed concurrent linear logic programming", Theoretical Computer Science. to appear.

[8] N. Kobayashi and A. Yonezawa. "Asynchronous communication model based on linear logic", Formal Aspects of Computing, Springer-Verlag, 7(2):113-149, 1995.

[9] J. Riely and M. Hennessy. "Distributed processes and location failures", Technical Report 2/97, Department of Computer Science, University of Sussex, 1997. 